\title{
Fast Multi-Baseline InSAR Phase Ambiguity Resolving Method with Low Computational Complexity ${ }^{*}$
}

\author{
Ming Li ${ }^{1,2, *}$, Haiping Wei ${ }^{1,2}$, Liping Xiao ${ }^{1,2}$, Zhiyong Suo ${ }^{3}$ \\ ${ }^{1}$ Beijing Aerospace Automatic Control Institute, Beijing 100854, China \\ ${ }^{2}$ National Key Laboratory of Science and Technology on Aerospace Intelligent Control, Beijing 100854, China \\ ${ }^{3}$ National Lab. of Radar Signal Processing, Xidian Univ., Xi'an, ShaanXi Province, 710071, China \\ Liming_up@163.com,whp_cara@163.com,xlp027@sina.com, zysuo@xidian.edu.cn
}

\begin{abstract}
A fast multi-baseline Interferometric synthetic aperture radar (InSAR) phase ambiguity resolving method is presented. The ambiguity solution vector of the shortest baseline is first computed and used as a reference, and then the ambiguity solutions of all the other baselines are selected with logical judgment of phase difference between the measured phase and the wrapped phase, which is obtained by rewrapping the product of the referenced phase and the ratios of the other baselines to the referenced baseline. 1-dimensional searching is then executed on the same index of the reserved ambiguity solutions vectors. Furthermore, the wrong estimates affected by noise are eliminated by taking the largest frequency of the ambiguity numbers counted in a window. Compared to the conventional Chinese remainder theory (CRT), the proposed method is more robust and has much less computational complexity. The validity is investigated with simulated results.

Index Terms - multi-baseline interferometric synthetic aperture radar (InSAR), phase ambiguity resolving, Chinese remainder theory (CRT)
\end{abstract}

\section{Introduction}

Multi-baseline Interferometric synthetic aperture radar (InSAR) is a powerful technique to obtain the digital elevation model (DEM) of complicated terrain[1,2]. Ideally, the Chinese remainder theory (CRT) is an effective method to resolve the multi-baseline InSAR phase ambiguities [3], but the rigorous stipulation is that all the baselines are co-prime to each other. For the conventional CRT, the optimal solution is obtained by multi-dimensional searching, i.e., if there are baselines coprime to each other, then loops must be used to achieve phase ambiguity resolving. For large scale multi-baseline InSAR phase unwrapping, the computational complexity is burdensome. In [4]-[6], the efficiency and robustness of CRT has been greatly improved with some given conditions. While for the multi-baseline InSAR, the conditions can not be perfectly met frequently. For example, the baseline length may be a negative real number, or the ambiguity number is not always a positive integer, or the moduli are not co-prime, etc. Considering the complicated factors in practical InSAR processing mentioned above, a multi-baseline InSAR phase ambiguity resolving method with low computational complexity is presented in this letter. We first take the ambiguity solution vector of the shortest baseline as a reference, and then the ambiguity solution vectors of all the other baselines are determined with logical judgment of the

\footnotetext{
* This work is supported by NSFC under grant No. 41001282.
}

phase difference between the measured phase and the wrapped phase, which is obtained by rewrapping the product of the referenced phase and the ratios of the other baselines to the referenced baseline, so the ambiguity vector (constructed by the reasonable ambiguity solution) length of each baseline is the same as the one of the referenced baseline. Then the ambiguity solution is obtained by searching the same index of the ambiguity vectors of all baselines under least square criterion.

\section{Multi-baseline InSAR Phase Ambiguity Resolving Method}

The relationship between the terrain height and the flattened InSAR phase is:

$$
h=a \psi / 2 \pi .
$$

where $a=\lambda r \sin \theta /(2 B)$ is the ambiguity height, $\lambda$ is the wavelength, $r$ is the slant range, $\theta$ is the radar look angle, $B$ is the baseline length ( $B$ may be a negative number determined by the relative position of the antennas), $\psi=\varphi+2 n \pi$ is the unwrapped phase, $n$ is the ambiguity number and $\varphi$ is the measured InSAR phase.

For the multi-baseline InSAR system, we have:

$$
\frac{2 n_{1} \pi+\varphi_{1}}{B_{1}}=\frac{2 n_{2} \pi+\varphi_{2}}{B_{2}}=\cdots=\frac{2 n_{N} \pi+\varphi_{N}}{B_{N}} .
$$

where $B_{i}(i=1,2, \cdots, N)$ is the length of baseline $i, n_{i}$ is the actual ambiguity number of $B_{i}, n_{i} \in\left[0, n_{i \max }\right], n_{i \max }$ is the maximal ambiguity number of the interferogram generated by $B_{i}$, and $\varphi_{i}$ is the measured phase of $B_{i}$.

According to (1) and (2), the congruence expressions of the multi-baseline InSAR can be defined as:

$$
\left\{\begin{array}{c}
\psi_{1}=b_{1}\left(2 n_{1} \pi+\varphi_{1}\right) \\
\psi_{2}=b_{2}\left(2 n_{2} \pi+\varphi_{2}\right) \\
\vdots \\
\psi_{N}=b_{N}\left(2 n_{N} \pi+\varphi_{N}\right)
\end{array} .\right.
$$


where $b_{i}=B_{1} / B_{i}, \psi_{i}$ is the unwrapped phase of $B_{i}$. To solve (3), we define the cost function as:

$$
\varepsilon=\sum_{i=1}^{N-1} \sum_{j=i+1}^{N}\left(\psi_{i}-\psi_{j}\right)^{2}
$$

The minimum $\varepsilon$ corresponds to the estimate of the ambiguity number set $\left(n_{1}, n_{2}, \cdots, n_{N}\right)$ of all baselines. When the number of baselines, $N$, increases, the computational work loads will increase rapidly for the conventional CRT method. Analyzing (3) and (4), we find that the differences between most of the ambiguity values are large, so it is redundant on minimizing $\varepsilon$ with those ambiguity values included, which is the reason that the conventional CRT is inefficient in multidimensional optimization.

Considering the shortcomings of the conventional CRT, a multi-baseline InSAR phase ambiguity resolving method based on 1-dimensional searching is presented. The detailed description of the method to estimate the ambiguity number of a pixel is given below:

Step1: Take the interferometric phase of the shortest baseline (marked as $B_{1}$ ) as a reference and form the ambiguity solution vector with $n_{1}\left(n_{1} \in\left[0, n_{1 \max }\right]\right)$.

Step2: For each ambiguity solution of $n_{1}\left(n_{1} \in\left[0, n_{1 \max }\right]\right)$, compute the reasonable ambiguity solution of the other baselines by (2).

The detailed description to compute the reasonable ambiguity solution with logical judgment is given below. From Step1, we take the interferogram of baseline $B_{1}$ as a reference. To determine the ambiguity number of each baseline $B_{i}$ corresponding to each ambiguity number of baseline $B_{1}$, the proportion between $B_{i}$ and $B_{1}$ (i.e., $b_{i}$ in (3)) is first used to compute the reasonable ambiguity solutions of baseline $B_{i}$, i.e.,

$$
\psi_{i}^{\prime}=\left(2 n_{1} \pi+\varphi_{1}\right) / b_{i} \text { with } n_{1} \in\left[0, n_{1 \max }\right] .
$$

Then the phase difference between the measured phase $\varphi_{i}$ and the wrapped phase of $\psi_{i}^{\prime}$. And then the ambiguity number of the current pixel is determined with logical judgment of the phase difference. If the absolute value of phase difference is smaller than $\pi$, the ambiguity number equals to the integer part of $\psi_{i}^{\prime} / 2 \pi$, or else the ambiguity number equals to the integer part of $\psi_{i}^{\prime} / 2 \pi+1$.

After step 2, the ambiguity solution vector length of each baseline equals to the one of baseline $B_{1}$, i.e., the maximal ambiguity solution vector length is $n_{1 \max }$.

Step3: By searching the same index of the ambiguity solution vectors of all baselines under least square criterion, the index with minimum value of $\varepsilon$ is selected and the ambiguity number set $\left(n_{1}, n_{2}, \cdots, n_{N}\right)$ corresponds to the current index is the optimal ambiguity solution. For all the pixels in the interferogram, Step1 Step3 is used iteratively.

Step4: To eliminate the wrong estimates affected by the noise, a window is used to slide on the ambiguity number estimates. And then the frequencies of the estimated ambiguity numbers in the local window are counted. The ambiguity number with the largest frequency is taken as the final estimation of the centric pixel of the local window. If phase noise filtering is executed before phase ambiguity estimation, step 4 can be omitted.

\section{Performance Investigation}

\section{A. Simulation results}

A three-baseline InSAR system is taken as an example. The parameters are listed in Table I.

TABLE I Simulation Parameters

\begin{tabular}{|c|c|}
\hline Wavelength & $0.03125(\mathrm{~m})$ \\
\hline Range Resolution & $2.5(\mathrm{~m})$ \\
\hline Baseline $B_{1}$ & $-63.8(\mathrm{~m})$ \\
\hline Baseline $B_{2}$ & $281.46(\mathrm{~m})$ \\
\hline Baseline $B_{3}$ & $345.27(\mathrm{~m})$ \\
\hline
\end{tabular}

The simulated interferograms are shown in Fig.1. Fig.1 (a) $\sim$ Fig.1(c) are the simulated noisy interferograms corresponding to baselines $B_{1}, B_{2}$ and $B_{3}$ respectively. According to the fringes in Fig.1, we know that the sign (determined by the relative position of two antennae) of $B_{1}$ is opposite to the sign of $B_{2}$ and $B_{3}$.

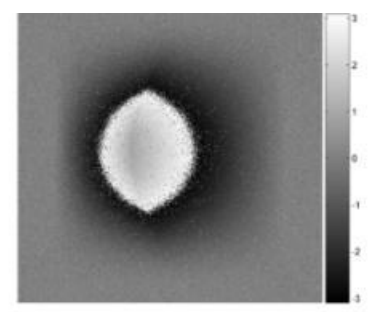

(a) Interferogram of baseline $B_{1}$

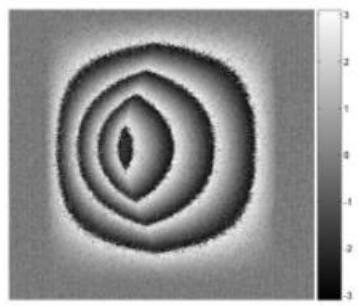

(b) Interferogram of baseline $B_{2}$

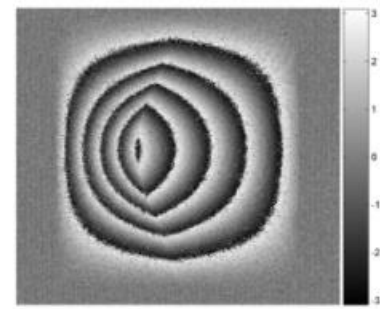

(c) Interferogram of baseline $B_{3}$
Fig.1 Simulated interferograms of different baselines

Fig. 2 is the ideal ambiguity numbers of different baselines. Fig.2 (a) Fig.2 (c) are the ambiguity numbers correspond to $B_{1}, B_{2}$ and $B_{3}$ respectively.

Fig. 3 shows the estimated ambiguity numbers of Fig.1 by using the method described above without wrong estimates 
elimination. Fig.3 (a) Fig.3 (c) are the primary estimated ambiguity numbers correspond to $B_{1}, B_{2}$ and $B_{3}$ respectively.

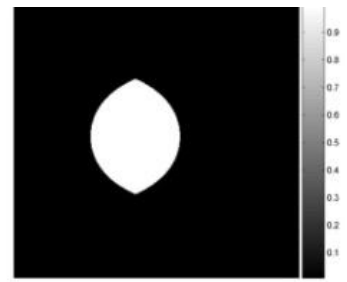

(a) Ideal ambiguity number of baseline $B$
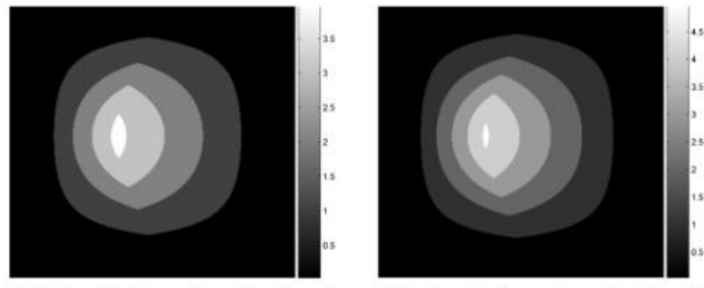

$\begin{array}{lll}\text { (b) Ideal ambiguity number of baseline } B_{2} & \text { (c) Ideal ambiguity number of baseline } B_{1}\end{array}$

Fig. 2 Ideal ambiguity numbers of different baselines

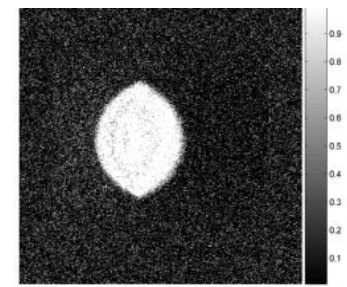

(a) Primary ambiguity number of baseline $B_{1}$
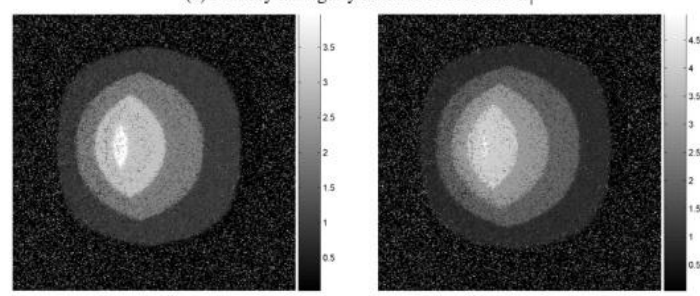

$\begin{array}{lll}\text { (b) Primary ambiguity number of baseline } B_{2} & \text { (c) Primary ambiguity number of baseline } B_{3}\end{array}$

Fig.3 Primary estimated ambiguity numbers of different baselines

Fig.4 shows the estimated ambiguity numbers of Fig. 1 by counting the ambiguity number frequency in an $11 \times 11$ window. Fig.4 (a) Fig.4 (c) are the final estimated ambiguity numbers correspond to $B_{1}, B_{2}$ and $B_{3}$ respectively.

From the simulation results, we can see that the presented method can successfully resolve the ambiguity number of interferograms obtained by the multi-baseline InSAR, even though in noisy environment and there are negative ambiguity numbers.

\section{B. Computational complexity analysis}

To solve (4), the computational complexity of the proposed 1-dimensional searching algorithm for one pixel includes $3 N\left(n_{1 \max }+1\right)(1+(N-1) / 2)$ multiplications and $N(N+1)\left(n_{1 \max }+1\right)$ additions. The computational complexity of $N$-dimensional searching of the conventional CRT includes
$\sum_{i=1}^{N}\left(n_{i \max }+1\right)+\frac{3 N(N-1)}{2} \prod_{i=1}^{N}\left(n_{i \max }+1\right) \quad$ multiplications $\quad$ and $\sum_{i=1}^{N}\left(n_{i \max }+1\right)+N(N-1) \prod_{i=1}^{N}\left(n_{i \max }+1\right) \quad$ additions. $\quad$ The computational efficiency of the proposed approach is approximately $\prod_{i=2}^{N}\left(n_{i \max }+1\right)$ times of the conventional CRT.

From the analysis of computational complexity above, the proposed method is more practical than the conventional CRT in multi-baseline InSAR processing.

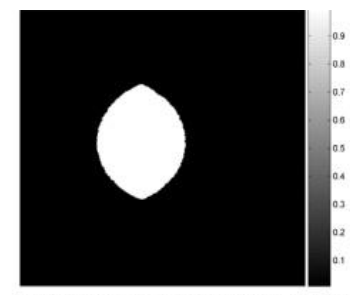

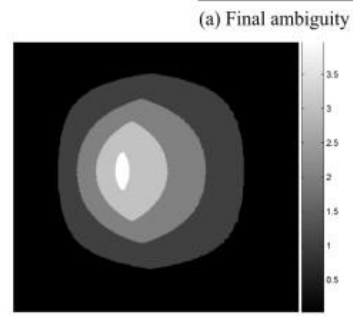

(b) Final ambiguity number of baseline $B_{2}$

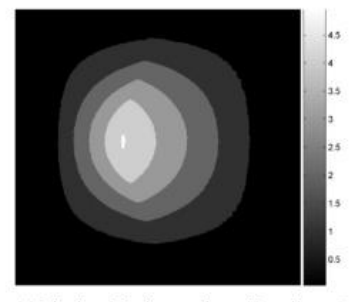

(c) Final ambiguity number of baseline $B_{3}$
Fig.4 Final estimated ambiguity numbers of different baselines

\section{Conclusion}

A multi-baseline InSAR phase ambiguity resolving method based on 1-dimesional searching under least square criterion is presented. The effect of noise on ambiguity resolving is eliminated by the statistical histogram of the ambiguity numbers in a local window. The computational burden is greatly alleviated compared to the conventional CRT method. The proposed method can also be used to the multifrequency InSAR phase ambiguity resolving.

\section{References}

[1] G. Fomaro, A. Monti Guarnieri, A. Pauciullo and Fo. De-Zan, "Maximum likelihood multi-baseline SAR interferometry," IEEE Proc. Radar Sonar Navig., 2006, 153(3): 279-288.

[2] F. K. Li and R. M. Goldstein, "Studies of multibaseline spaceborne interferometric synthetic aperture radars," IEEE Trans. on Geoscience and Remote Sensing. 1990, 28(1):88-97.

[3] W. Xu, E. Chang, L. Kwoh, H. Lim, and W. Cheng, "Phase-unwrapping of SAR interferogram with multi-frequency or multi-baseline," in Proc. IEEE Int. Geosci. Remote Sens. Symp., Pasadena, CA, 1994, pp. 730732.

[4] X. Xia and G. Wang, "Phase unwrapping and a robust Chinese remainder theorem," IEEE Signal Processing Letters, 2007, 14(4): 247-250.

[5] X. Li and X. Xia, "A fast robust chinese remainder theorem based phase unwrapping algorithm," IEEE Signal Processing Letters, 2008, 15: 665668.

[6] W. Wang and X. Xia, "A closed-form robust chinese remainder theorem and its performance analysis," IEEE Trans. on Signal Processing, 2010, 58(11): 5655-5666. 